\title{
Randomized phase II study of sequential carboplatin plus paclitaxel and gefitinib in chemotherapy-naïve patients with advanced or metastatic non-small-cell lung cancer: Long-term follow-up results
}

\author{
EMI KUBO, NOBORU YAMAMOTO, HIROSHI NOKIHARA, YUTAKA FUJIWARA, \\ HIDEHITO HORINOUCHI, SHINTARO KANDA, YASUSHI GOTO and YUICHIRO OHE \\ Department of Thoracic Oncology, National Cancer Center Hospital, Tokyo 104-0045, Japan
}

Received December 21, 2015; Accepted August 12, 2016

DOI: $10.3892 / \mathrm{mco} .2016 .1076$

\begin{abstract}
The epidermal growth factor receptor (EGFR) tyrosine kinase inhibitor gefitinib was initially approved in Japan in 2002 for the treatment of advanced or metastatic non-small-cell lung cancer (NSCLC); however, the optimal order of conventional cytotoxic chemotherapy (carboplatin and paclitaxel) and gefitinib administration has not been determined. We conducted a randomized phase II study of carboplatin and paclitaxel followed by gefitinib vs. gefitinib followed by carboplatin and paclitaxel to select a candidate for further development in a phase III study of chemotherapy-naïve patients with advanced or metastatic NSCLC, regardless of their EGFR mutation status. A total of 97 patients meeting this description were randomly assigned to arm A (carboplatin and paclitaxel followed by gefitinib; $n=49$ ) or B (gefitinib followed by carboplatin and paclitaxel; $n=48$ ) from June, 2003 to October, 2005. Carboplatin and paclitaxel were administered in 4 cycles every 3 weeks; gefitinib was continued until disease progression or development of unacceptable toxicity. The primary endpoint was overall survival; the secondary endpoints were response rate and adverse event prevalence. The median overall follow-up was 65.1 months (range, 28.7-75.1 months). The major toxicities were hematological (carboplatin and paclitaxel) or skin rash, diarrhea and hepatic dysfunction (gefitinib). Interstitial lung disease was observed in 1 patient from each arm. In arms A and B, the carboplatin and paclitaxel response rate, gefitinib response rate, and median survival durations were 34.8 and $26.5 \%$, 33.3 and $35.7 \%$, and 18.8 and 17.2 months, respectively. Arm A was selected for a subsequent phase III study.
\end{abstract}

Correspondence to: Dr Noboru Yamamoto, Department of Thoracic Oncology, National Cancer Center Hospital, Tsukiji 5-1-1, Chuo-ku, Tokyo 104-0045, Japan

E-mail: nbryamam@ncc.go.jp

Key words: gefitinib, carboplatin, paclitaxel, non-small-cell lung cancer, epidermal growth factor receptor gene mutation

\section{Introduction}

Lung cancer is the leading cause of malignancy-related deaths worldwide, and non-small-cell lung cancer (NSCLC) accounts for $\sim 80 \%$ of all types of lung cancer (1). Approximately two-thirds of NSCLC cases present as locally advanced or metastatic disease, and systemic chemotherapy, including molecular-targeted therapy, plays a key role in the treatment of such patients. However, the prognosis of such cases remains poor; thus, there is a pressing need for more effective, tolerable and individualized treatments to improve clinical outcome.

In the 2000s, anticancer agents were administered to patients with advanced or metastatic NSCLC, regardless of their specific biological characteristics, in an 'unselected' manner. At that time, platinum-based chemotherapies, including carboplatin and paclitaxel, were widely recognized as standard treatments for advanced or metastatic NSCLC. In previous randomized phase III studies, the response rates to a combination of carboplatin and paclitaxel ranged from 17 to $32.4 \%$, with median survival times (MST) of 8.1-12.3 months $(2,3)$.

In 2002, the first molecular-targeted agent, gefitinib, was approved for advanced or metastatic NSCLC in Japan, prior to its approval in other countries. However, predictive biomarkers, such as mutations in the gene encoding epidermal growth factor receptor (EGFR) had not been identified, and the response rates among unselected patients ranged from 12 to $18 \%$, with MSTs of 7.0-7.6 months, in earlier phase II studies (IDEAL 1 and 2) $(4,5)$. However, the discovery of EGFR mutations as predictive biomarkers of an antitumor response to gefitinib greatly elucidated the indication for gefitinib treatment in patients with advanced or metastatic NSCLC (6,7). The response rates to gefitinib among $E G F R$-mutant patients were $70 \%(8,9)$ and gefitinib, as well as erlotinib and afatinib, are currently established as standard first-line treatment for patients with advanced or metastatic NSCLC harboring EGFR mutations.

Although the combination of carboplatin and paclitaxel, as well as gefitinib, were considered to be effective for the treatment of advanced or metastatic NSCLC, the optimal order of these two treatments had not been determined by 2002 . Accordingly, from 2003 to 2005, we conducted a randomized phase II study to compare a combination of carboplatin and 
paclitaxel followed by gefitinib monotherapy, with gefitinib monotherapy followed by a combination of carboplatin and paclitaxel in chemotherapy-naïve patients with advanced or metastatic NSCLC. The objective of this study was to select a candidate arm for further investigation. After obtaining long-term follow-up data, we herein report the final analysis of this study.

\section{Patients and methods}

Patient selection. Patients with histologically and/or cytologically confirmed NSCLC were eligible for this study. The eligibility criteria were as follows: Clinical stage IV or IIIB (including only patients without indications for curative radiotherapy, such as malignant pleural effusion or dissemination, malignant pericardiac effusion, or metastatic lesion in the same lobe as the primary lesion) based on the fifth Union for International Cancer Control TNM classification (http://www. uicc.org/resources/tnm); no prior systemic chemotherapy; no prior surgery and/or radiotherapy at the primary site; age 20-74 years; Eastern Cooperative Oncology Group performance status (ECOG-PS) of 0 or 1; at least 1 measurable lesion $\geq 1 \mathrm{~cm}$; and adequate organ function (white blood cell count $\geq 4,000 / \mathrm{mm}^{3}$, neutrophil count $\geq 2,000 / \mathrm{mm}^{3}$, hemoglobin level $\geq 9.5 \mathrm{~g} / \mathrm{dl}$, platelet count $\geq 100,000 / \mathrm{mm}^{3}$, serum creatinine level $\leq 1.5 \mathrm{mg} / \mathrm{dl}$, aspartate aminotransferase level $\leq 2$ times the upper limit of normal, alanine aminotransferase level $\leq 2$ times the upper limit of normal, total bilirubin level $\leq 1.5 \mathrm{mg} / \mathrm{dl}$ and partial arterial oxygen pressure $\geq 60$ Torr). The exclusion criteria were as follows: Serious concomitant illness (severe heart disease, uncontrolled diabetes mellitus, uncontrolled hypertension, or active infection); obvious interstitial pneumonia or pulmonary fibrosis on chest X-ray; pericardial or pleural effusion requiring drainage; synchronous active malignancy; severe drug allergy; and pregnancy or breastfeeding. Patients with brain metastases were eligible for enrollment if they were asymptomatic and did not require steroid or anticonvulsant therapy.

Written informed consent was obtained from all the participating patients and the study protocol was approved by the Institutional Review Board of the National Cancer Center (Tokyo, Japan).

Study design. The aim of this randomized phase II study aimed to select a candidate arm for further development in a phase III study by investigating the optimal order of cytotoxic chemotherapy (carboplatin and paclitaxel) and molecular-targeted therapy (gefitinib) administration in chemotherapy-naïve patients with advanced or metastatic NSCLC. The primary endpoint was overall survival, and the secondary endpoints were response rate and prevalence of adverse events. The patients were randomly assigned in a 1:1 manner to either arm A (carboplatin and paclitaxel followed by gefitinib) or arm B (gefitinib followed by carboplatin plus paclitaxel) (Fig. 1). Random assignment was stratified according to gender (female vs. male) and histology (adenocarcinoma vs. others).

Treatment. The treatment plan for arm A included 4 cycles of carboplatin and paclitaxel at 3-week intervals, followed by gefitinib monotherapy. Gefitinib monotherapy was administered from day 22 to day 50, or after the fourth cycle of carboplatin and paclitaxel, even if the patients had stable disease. If patients in arm A experienced disease progression or unacceptable toxicities before the 4 cycles of treatment were completed, gefitinib monotherapy was subsequently initiated within the 4-week drug holiday. Gefitinib monotherapy was continued until development of disease progression or unacceptable toxicity. Arm B patients received gefitinib monotherapy, which continued until disease progression or development of unacceptable toxicity, followed by 4 cycles of carboplatin and paclitaxel at 3-week intervals.

In both arms, paclitaxel was administered intravenously on day 1 at a dose of $200 \mathrm{mg} / \mathrm{m}^{2}$ over a 3-h period, followed by carboplatin at a dose calculated to produce an area under the concentration-time curve of $6.0 \mathrm{mg} / \mathrm{min} / \mathrm{ml}$. Gefitinib was orally administered at a once-daily dose of $250 \mathrm{mg}$. Subsequent treatments were not restricted in either arm of this study.

Study evaluations. The pretreatment evaluation included a complete medical history, physical examination, complete and differential blood cell counts, routine chemistry measurement, urinalysis, chest radiography, chest computed tomography (CT) scan, brain CT or magnetic resonance imaging, abdominal ultrasonography and/or CT, and electrocardiogram. Complete and differential blood cell counts and routine chemistry measurements were performed at least weekly during the first carboplatin and paclitaxel cycle, or within 4 weeks after the initiation of gefitinib monotherapy. A chest radiograph was obtained weekly during the first carboplatin and paclitaxel cycle or gefitinib monotherapy to evaluate potential pulmonary toxicity, and as needed thereafter.

The overall survival duration was assessed from the date of registration to the date of death from any cause. If death did not occur, data were censored based on the last date the patient was known to be alive.

The objective tumor response was evaluated in all eligible patients according to the Response Evaluation Criteria in Solid Tumors (10). Toxicities were graded according to the National Cancer Institute Common Toxicity Criteria (NCI-CTC), version 2.0 (http://ctep.cancer.gov/protocolDevelopment/electronic_applications/ctc.htm).

Evaluation of EGFR mutation status. The EGFR mutation status was analyzed in 41 patients with available tumor samples. DNA was extracted from archived Papanicolaou-stained cytological slides and/or formalin-fixed, paraffin-embedded (FFPE) tissues using the QIAamp DNA Micro kit (Qiagen, Venlo, The Netherlands). The two major hotspots for EGFR mutations, an in-frame deletion including amino acids at codons 747-749 (DEL) in exon 19 and a missense mutation at codon 858 (L858R) in exon 21, were analyzed via high-resolution melting analysis, as previously described $(11,12)$. Polymerase chain reaction was performed using primers designed to amplify a region containing E746-I759 or L858 of EGFR and LC Green Idye (Roche Diagnostics, Indianapolis, IN, USA). Melting curves were obtained using HR-1 (Idaho Technology, Salt Lake City, UT, USA) and the curves produced for samples and controls were compared.

Statistical analysis. As overall survival was the primary endpoint of this study, the required sample size was calculated 


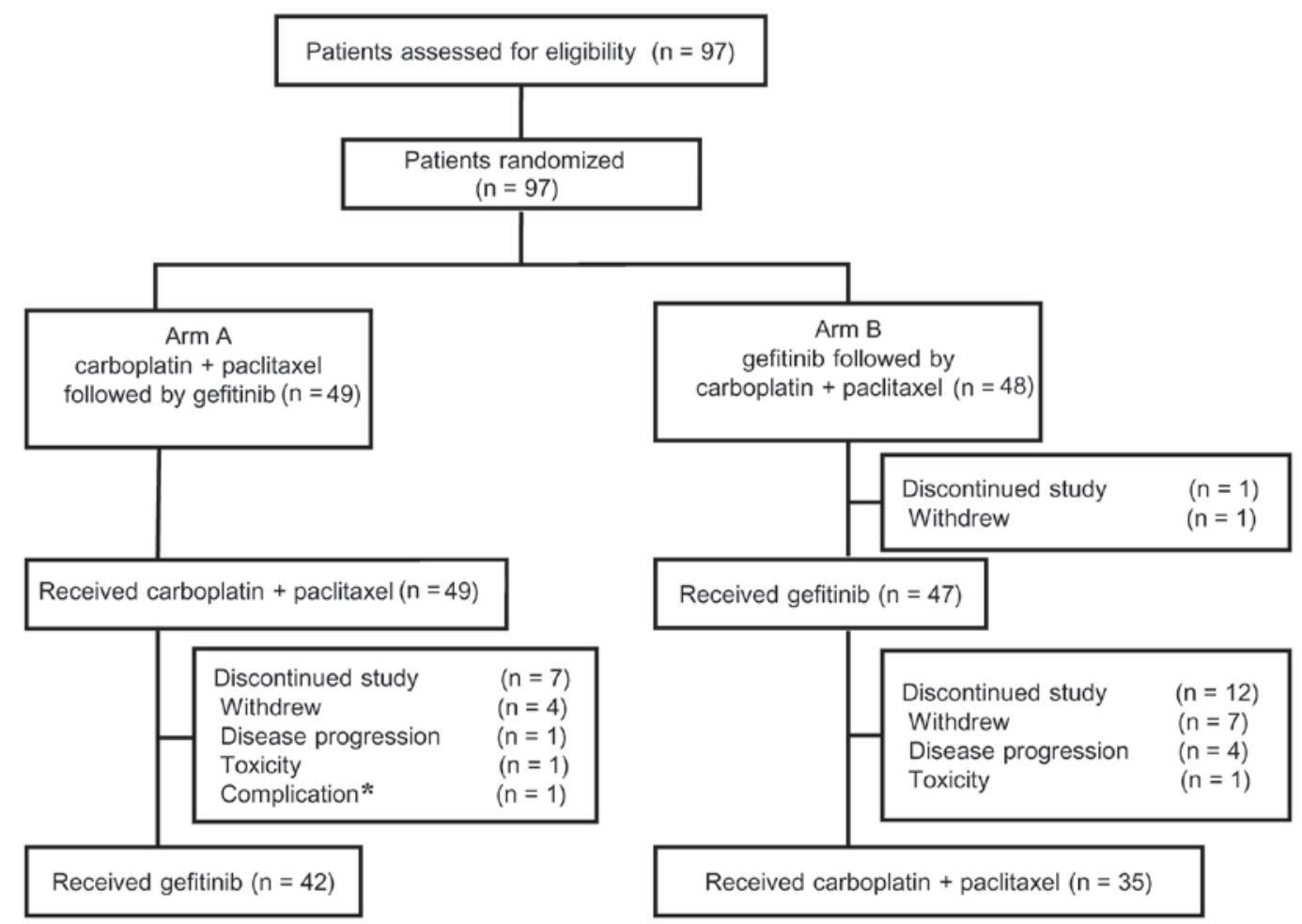

Figure 1. CONSORT diagram of the present study. *Pneumonitis after palliative radiotherapy.

according to the selection designs of survival-based pilot studies (13). The required total sample size was determined to be 87 with the following assumptions: i) The survival curve was exponential; ii) the MST of the worse arm was 9 months and that of the better arm was 9 months $\mathrm{x} 1.4$; iii) the correct selection probability was $90 \%$; and iv) the additional follow-up (in years) after the end of accrual was 1 year. After assuming a $10 \%$ non-evaluable rate, a total of 96 patients and an accrual period of 3 years were required to evaluate this study. The survival distribution was estimated using the Kaplan-Meier method.

A two-sided P-value of $\leq 0.05$ was considered to indicate statistically significant differences. All statistical analyses were performed using JMP software, version 11.2 (SAS Institute, Inc., Cary, NC, USA).

\section{Results}

Patient characteristics. A total of 97 patients were enrolled between June, 2003 and October, 2005, of whom 49 were randomly assigned to arm A (carboplatin and paclitaxel followed by gefitinib) and 48 to arm B (gefitinib followed by carboplatin and paclitaxel). The CONSORT diagram of this study is shown in Fig. 1. The patient characteristics were well balanced between the two treatment arms, with median ages of 63 (range, 32-74) and 61 (range, 45-73) years, male/female ratios of 28/21 and 28/20, ECOG-PS 0/1 ratios of 23/26 and 24/24, and stage IIIB/IV ratios of 11/38 and 11/37 in arms A and B, respectively (Table I). A total of $85(88 \%)$ patients were diagnosed with adenocarcinoma. One patient in arm B withdrew consent and, therefore, did not receive the study treatment (gefitinib). The remaining 96 patients were evaluable for toxicity and antitumor response following first-line treatment (i.e., carboplatin and paclitaxel in arm A, gefitinib in arm B) and overall survival.

Administered treatment. In arm A, 49 patients received carboplatin and paclitaxel, with a median number of 4 (range, 1-5) treatment cycles, and 42 transitioned to gefitinib monotherapy (Fig. 1). The main reasons for transitioning to gefitinib monotherapy were progressive disease (PD; $n=34$ ) and toxicity $(n=8)$. A total of 7 patients did not receive gefitinib monotherapy due to the following reasons: Consent withdrawal, $(n=4)$; disease progression $(n=1)$; pneumonitis after palliative radiotherapy $(n=1)$; and prolonged toxicity from carboplatin and paclitaxel $(n=1)$. The median duration of gefitinib monotherapy was 73 days (range, 46-154 days).

In arm B, 47 of 48 patients received gefitinib monotherapy, and 35 of 47 patients transitioned to carboplatin and paclitaxel, with a median of 4 (range, 1-4) treatment cycles. The median gefitinib monotherapy duration was 87 days (range, 34-137 days). The main reasons for transitioning to carboplatin and paclitaxel were PD $(n=32)$ and toxicity $(n=3)$. Of the 47 patients, 12 did not transition to carboplatin and paclitaxel treatment due to the following reasons: Consent withdrawal $(n=7) ;$ PD $(n=4)$; and toxicity $(n=1)$.

Efficacy. The response rates to carboplatin and paclitaxel were $34.8 \%$ [95\% confidence interval (CI): 21.0-48.5] and $26.5 \%$ (95\% CI: 11.6-41.3) in arms A and B, respectively (Table II). The response rate to gefitinib was $35.7 \%$ (95\% CI: 18.0-53.5) and $33.3 \%$ (95\% CI: 19.6-47.1) in arm A and B, respectively. The rate did not differ significantly between the treatment arms.

The median overall follow-up duration was 65.1 months (range, 28.7-75.1 months). The median progression-free survival 
Table I. Patient characteristics.

\begin{tabular}{|c|c|c|}
\hline Characteristics & $\begin{array}{c}\text { Arm A }(\mathrm{n}=49) \\
\text { No. }(\%)\end{array}$ & $\begin{array}{c}\text { Arm B }(n=48) \\
\text { No. }(\%)\end{array}$ \\
\hline \multicolumn{3}{|l|}{ Age, years } \\
\hline Median (range) & $63(32-74)$ & $61(45-73)$ \\
\hline \multicolumn{3}{|l|}{ Gender } \\
\hline Male & $28(57.1)$ & $28(58.3)$ \\
\hline Female & $21(42.9)$ & $20(41.7)$ \\
\hline \multicolumn{3}{|l|}{ ECOG PS } \\
\hline 0 & $23(46.9)$ & $24(50.0)$ \\
\hline 1 & $26(53.1)$ & $24(50.0)$ \\
\hline \multicolumn{3}{|l|}{ Histology } \\
\hline Adenocarcinoma & $43(87.8)$ & $42(87.5)$ \\
\hline Squamous cell & $2(4.1)$ & $1(2.1)$ \\
\hline $\mathrm{NSCLC}^{\mathrm{a}}$ & $4(8.2)$ & $5(10.4)$ \\
\hline \multicolumn{3}{|l|}{ Stage } \\
\hline IIIB & $11(22.4)$ & $11(22.9)$ \\
\hline IV & $38(77.6)$ & $37(77.1)$ \\
\hline \multicolumn{3}{|l|}{ Smoking status } \\
\hline Current and/or past & $27(55.1)$ & $28(58.3)$ \\
\hline Never & $22(44.9)$ & $20(41.7)$ \\
\hline \multicolumn{3}{|l|}{ Previous treatment } \\
\hline No & $38(77.6)$ & $39(81.3)$ \\
\hline Yes & $11(22.4)$ & $9(18.8)$ \\
\hline Radiotherapy for bone & 2 & 4 \\
\hline Radiotherapy for brain & 3 & 2 \\
\hline Surgery for brain & 0 & 1 \\
\hline Drainage & 6 & 4 \\
\hline
\end{tabular}

${ }^{a}$ Not further characterized. ECOG, Eastern Cooperative Oncology Group; PS, performance status; NSCLC, non-small-cell lung cancer.

durations with gefitinib monotherapy were 2.4 (95\% CI: $1.5-5.1)$ and 2.9 (95\% CI: 1.1-4.6) months in arms A and B, respectively; the difference was not statistically significant.

At the time of the most recent update (February 17, 2015), 91 of the 97 patients had succumbed to the disease, and 6 had been lost to follow-up. The MSTs were 19.1 (95\% CI: 11.7-26.0) and 18.2 (95\% CI: 13.5-21.6) months in arms $\mathrm{A}$ and $\mathrm{B}$, respectively $(\mathrm{P}=0.971 ;$ Fig. 2$)$. The respective 1- and 2-year survival rates were $61.2 \%$ (95\% CI: 47.0-73.7) and $42.8 \%(95 \%$ CI: 29.9-56.9) in arm A, and $68.7 \%$ (95\% CI: $54.4-80.1)$ and $31.3 \%$ (95\% CI: 19.8-45.6) in arm B (Fig. 2). The treatment arms did not significantly differ with respect to the MSTs or 1- and 2-year survival rates.

Toxicity. The toxicity profiles of the two treatment arms are shown in Table III. In both arms, the major toxicities associated with carboplatin and paclitaxel were neutropenia, leukopenia, peripheral neuropathy, arthralgia and nausea, whereas diarrhea, rash, and hepatic dysfunction were associated with gefitinib. The frequency and severity of these toxicities did not differ significantly between the treatment arms.
Table II. Response to treatment.

A, Response to first treatment

\begin{tabular}{|c|c|c|}
\hline & $\operatorname{Arm~A~}(n=49)$ & Arm B $(n=48)$ \\
\hline Type of response & Carboplatin+PTX & Gefitinib \\
\hline Assessable cases, $\mathrm{n}$ & 49 & 47 \\
\hline $\mathrm{CR}$ & 0 & 0 \\
\hline PR & 16 & 15 \\
\hline SD & 20 & 11 \\
\hline PD & 10 & 19 \\
\hline $\mathrm{NE}$ & 3 & 2 \\
\hline $\mathrm{RR}, \%(95 \% \mathrm{CI})$ & $34.8(21.0-48.5)$ & $33.3(19.6-47.1)$ \\
\hline
\end{tabular}

$\mathrm{B}$, Response to second treatment

\begin{tabular}{lcc}
\hline Type of response & Gefitinib & Carboplatin+PTX \\
\hline Assessable cases, $\mathrm{n}$ & 29 & 35 \\
CR & 0 & 1 \\
PR & 10 & 8 \\
SD & 5 & 17 \\
PD & 13 & 8 \\
NE & 1 & 1 \\
RR, \% (95\% CI $)$ & $35.7(18.0-53.5)$ & $26.5(11.6-41.3)$ \\
\hline
\end{tabular}

$\mathrm{CR}$, complete response; $\mathrm{PR}$, partial response; $\mathrm{SD}$, stable disease; $\mathrm{PD}$, progressive disease; NE, not evaluable; RR, response rate; PTX, paclitaxel; CI, confidence interval.

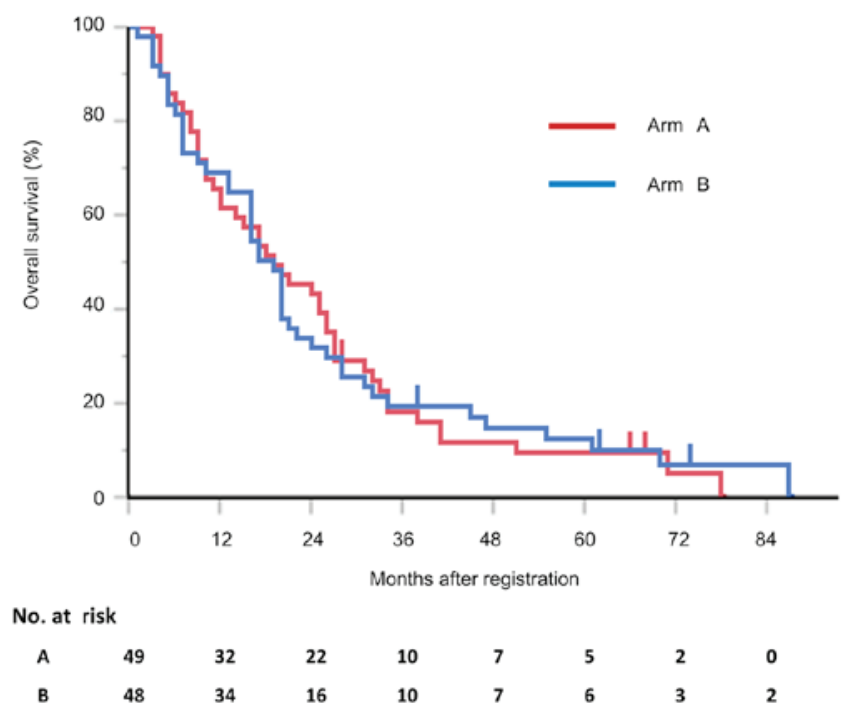

Figure 2. Kaplan-Meier estimates of overall survival after study registration. Arm A: Carboplatin and paclitaxel followed by gefitinib. Arm B: Gefitinib followed by carboplatin and paclitaxel.

Two patients experienced interstitial lung disease (ILD) in this study. One patient developed ILD on day 51 of gefitinib monotherapy in arm A, and this case was considered to be strongly associated with gefitinib. The other patient developed 
Table III. Toxicity profiles.

A, Carboplatin + paclitaxel

\begin{tabular}{|c|c|c|c|c|c|c|}
\hline \multirow[b]{3}{*}{ Toxicity } & \multicolumn{3}{|c|}{$\operatorname{Arm~A~}(n=49)$} & \multicolumn{3}{|c|}{ Arm B $(n=35)$} \\
\hline & \multicolumn{3}{|c|}{ Grade, n (\%) } & \multicolumn{3}{|c|}{ Grade, n (\%) } \\
\hline & $1-2$ & 3 & 4 & $1-2$ & 3 & 4 \\
\hline Leukopenia & $28(57)$ & $14(29)$ & $1(2)$ & $16(44)$ & $14(40)$ & $0(0)$ \\
\hline Neutropenia & $8(17)$ & $17(35)$ & $19(39)$ & $5(11)$ & $10(29)$ & $15(43)$ \\
\hline Thrombocytopenia & $24(49)$ & $6(12)$ & $0(0)$ & $16(45)$ & $8(22)$ & $0(0)$ \\
\hline Anemia & $40(82)$ & $5(10)$ & $1(2)$ & $23(63)$ & $9(26)$ & $2(6)$ \\
\hline AST increased & $25(51)$ & $1(2)$ & $0(0)$ & $18(51)$ & $1(3)$ & $0(0)$ \\
\hline ALT increased & $26(53)$ & $2(5)$ & $0(0)$ & $18(51)$ & $5(14)$ & $0(0)$ \\
\hline Bilirubin increased & $16(33)$ & $0(0)$ & $0(0)$ & $12(34)$ & $0(0)$ & $0(0)$ \\
\hline Nausea/vomiting & $23(46)$ & $2(5)$ & $0(0)$ & $22(62)$ & $0(0)$ & $0(0)$ \\
\hline Arthralgia/myalgia & $38(77)$ & $1(2)$ & $0(0)$ & $25(71)$ & $0(0)$ & $0(0)$ \\
\hline Neuropathy & $33(67)$ & $2(4)$ & $0(0)$ & $18(51)$ & $3(9)$ & $0(0)$ \\
\hline Infection & $6(12)$ & $0(0)$ & $0(0)$ & $7(20)$ & $0(0)$ & $0(0)$ \\
\hline Febrile neutropenia & $0(0)$ & $7(14)$ & $0(0)$ & $0(0)$ & $2(5)$ & $0(0)$ \\
\hline Interstitial lung disease & $0(0)$ & $0(0)$ & $0(0)$ & $0(0)$ & $1(2)$ & $0(0)$ \\
\hline
\end{tabular}

B, Gefitinib

\begin{tabular}{|c|c|c|c|c|c|c|}
\hline \multirow[b]{3}{*}{ Toxicity } & \multicolumn{3}{|c|}{$\operatorname{Arm~A~}(n=42)$} & \multicolumn{3}{|c|}{ Arm B $(n=47)$} \\
\hline & \multicolumn{3}{|c|}{ Grade, n (\%) } & \multicolumn{3}{|c|}{ Grade, n (\%) } \\
\hline & $1-2$ & 3 & 4 & $1-2$ & 3 & 4 \\
\hline AST increased & $12(28)$ & $4(9)$ & $0(0)$ & $22(46)$ & $4(8)$ & $0(0)$ \\
\hline ALT increased & $18(42)$ & $3(7)$ & $0(0)$ & $21(44)$ & $9(19)$ & $0(0)$ \\
\hline Bilirubin increased & $3(7)$ & $0(0)$ & $0(0)$ & $6(12)$ & $0(0)$ & $0(0)$ \\
\hline Nausea/vomiting & $6(14)$ & $0(0)$ & $0(0)$ & $4(8)$ & $1(2)$ & $0(0)$ \\
\hline Rash & $30(71)$ & $0(0)$ & $0(0)$ & $36(76)$ & $1(2)$ & $0(0)$ \\
\hline Dry skin & $6(14)$ & $0(0)$ & $0(0)$ & $4(8)$ & $0(0)$ & $0(0)$ \\
\hline Diarrhea & $20(47)$ & $0(0)$ & $0(0)$ & $22(55)$ & $0(0)$ & $0(0)$ \\
\hline Stomatitis & $4(9)$ & $0(0)$ & $0(0)$ & 9 (19) & $0(0)$ & $0(0)$ \\
\hline Infection & $4(9)$ & $0(0)$ & $0(0)$ & $1(2)$ & $0(0)$ & $0(0)$ \\
\hline Interstitial lung disease & $0(0)$ & $1(2)$ & $0(0)$ & $0(0)$ & $0(0)$ & $0(0)$ \\
\hline
\end{tabular}

AST, aspartate aminotransferase; ALT, alanine aminotransferase.

possible treatment-related ILD after the second cycle of carboplatin and paclitaxel in arm B. Both patients required steroid therapy to recover from ILD. The patient from arm B succumbed to perforative peritonitis on day 36 after initiating gefitinib monotherapy, although this event was not considered to be treatment-related, but rather due to tumor progression.

Exploratory analysis. FFPE samples suitable for mutation analysis were available from 41 of the 96 patients in this study (21 in arm A and 20 in arm B). EGFR mutations were detected in $13(61.9 \%)$ patients in arm A (9 in DEL and 4 in L858R), and $7(35 \%)$ in arm B (4 in DEL and 3 in L858R). Seven of the $13(53.8 \%)$ and 6 of the $7(85.7 \%)$ patients with EGFR mutations in arms A and B, respectively, achieved a partial response to gefitinib monotherapy. In both arms, the survival of patients harboring $E G F R$ mutations tended to be longer compared with that of patients without EGFR mutations, although these differences were not statistically significant (data not shown).

\section{Discussion}

The aim of this randomized phase II study was to determine the optimal order of cytotoxic chemotherapy (i.e., carboplatin 
and paclitaxel) and gefitinib administration for the treatment of NSCLC, regardless of the EGFR mutation status, for use in a subsequent phase III study comparing chemotherapy with the better regimen of this phase II study. In this study, overall survival did not differ significantly between the two treatment arms. The response rate to carboplatin and paclitaxel in both treatment arms (i.e., arm A + arm B) was $31.2 \%$ (95\% CI: 21.1-41.4), and the response rate to gefitinib was 34.2\% (95\% CI: 23.4-45.1). The major toxicities associated with carboplatin and paclitaxel were neutropenia and leukopenia, and their incidences were almost equal between the two treatment arms. The major toxicities associated with gefitinib were rash and liver dysfunction, which also occurred at nearly equal rates in the two treatment arms. We decided upon arm A (carboplatin and paclitaxel followed by gefitinib) as a candidate arm for a subsequent phase III study, as carboplatin and paclitaxel was an established first-line treatment for advanced NSCLC at the time of study planning.

Immediately after completing patient enrollment in our study, a large-scale Asian randomized phase III study of treatment for advanced NSCLC (IPASS) was launched (14). The patients in that study were selected using more strict clinical criteria (i.e., Asian population, adenocarcinoma only, and light or never-smokers). During patient enrollment for our randomized phase II study, we remained aware of the favorable clinical characteristics of patients who may benefit from gefitinib, specifically female patients with adenocarcinoma who were light or never-smokers. In our study, the proportions of patients with adenocarcinoma and never-smokers were higher compared with those in previous randomized phase III studies $(2,3)$. The IPASS study was conducted in a global setting, and our institute had joined this study; thus, we did not conduct our previously planned phase III study. From a different viewpoint, our randomized phase II study may be considered as a beginning stage of the IPASS study. In the IPASS study, the overall survival rates between the two treatment arms (carboplatin and paclitaxel followed by gefitinib vs. gefitinib followed by carboplatin and paclitaxel) did not differ significantly. However, progression-free survival was significantly better in the experimental arm (gefitinib followed by carboplatin and paclitaxel). By contrast, our study was not designed to assess progression-free survival between the two treatment arms, as subsequent gefitinib monotherapy was initiated in arm A following the completion of 4 cycles of carboplatin and paclitaxel, without confirmation of disease progression.

An activating somatic EGFR mutation was discovered in 2004 (6,7). After this landmark discovery, treatment strategies changed dramatically from phenotype- to genotype-based. Currently, the EGFR mutation status is initially determined from biopsy samples and used to determine the use of EGFR-TKIs. Accordingly, several randomized phase III studies have been conducted to investigate the most favorable order of cytotoxic chemotherapy and EGFR-TKI administration. The NEJ002 study compared carboplatin and paclitaxel followed by gefitinib with gefitinib followed by carboplatin and paclitaxel in patients with EGFR-mutant NSCLC, but failed to observe a significant difference in overall survival (9). Similarly, the WTJOG3405 study compared cisplatin and docetaxel followed by gefitinib with gefitinib followed by cisplatin and docetaxel in patients with EGFR-mutant NSCLC, and did not observe significantly different survival outcomes (8). Other global phase III studies (e.g., OPTIMAL and EURTAC) also compared cytotoxic chemotherapy and EGFR-TKI as first-line treatments for EGFR-mutant NSCLC $(15,16)$. Although overall survival did not differ between cytotoxic chemotherapy and EGFR-TKI in those studies, progression-free survival was significantly better in the EGFR-TKI arms of all studies (NEJ002, WTJOG3405, OPTIMAL and EURTAC). These reproducible and favorable outcomes indicate a progression-free survival advantage and higher response rate associated with EGFR-TKIs, contributing to the recommendation of these agents as first-line treatment for patients with EGFR-mutant NSCLC.

When we planned this randomized phase II study in 2002, favorable clinical pretreatment factors (i.e., female gender, adenocarcinoma and light or never-smokers) or the EGFR mutation status had not been clearly established with regard to patient selection for clinical studies incorporating EGFR-TKIs. Although the survival outcome in our study appears to be similar to that of the subsequent large-scale IPASS phase III study, we are unable to apply the results of our study into the current routine clinical practice. However, our results may also be considered an initial step toward a subsequent large-scale phase III study.

Currently, the individualization of EGFR-TKI treatment is becoming more precise. For example, the second-generation EGFR-TKI afatinib conferred a survival advantage over cytotoxic chemotherapy to patients with EGFR exon 19 deletion in an integrated survival analysis of Lux-Lung-3 and -6 (17). Moreover, global studies are investigating the ability of third-generation EGFR-TKIs to overcome the resistant EGFR-T790M mutation, and the approval of these agents is expected (18-20). In the near future, additional individualized treatments may be established for the treatment of EGFR-mutant NSCLC.

In conclusion, overall survival did not significantly differ between the two arms of our study in patients with advanced NSCLC, regardless of their EGFR mutation status; accordingly, we selected carboplatin and paclitaxel followed by gefitinib for a subsequent phase III study. Our study results may represent an important step toward a subsequent phase III study.

\section{References}

1. Torre LA, Bray F, Siegel RL, Ferlay J, Lortet-Tieulent J and Jemal A: Global cancer statistics, 2012. CA Cancer J Clin 65: 87-108, 2015

2. Ohe Y, Ohashi Y, Kubota K, Tamura T, Nakagawa K, Negoro S, Nishiwaki Y, Saijo N, Ariyoshi Y and Fukuoka M: Randomized phase III study of cisplatin plus irinotecan versus carboplatin plus paclitaxel, cisplatin plus gemcitabine and cisplatin plus vinorelbine for advanced non-small-cell lung cancer: Four-arm cooperative study in Japan. Ann Oncol 18: 317-323, 2007.

3. Schiller JH, Harrington D, Belani CP, Langer C, Sandler A, Krook J, Zhu J and Johnson DH; Eastern Cooperative Oncology Group: Comparison of four chemotherapy regimens for advanced non-small-cell lung cancer. N Engl J Med 346: 92-98, 2002.

4. Kris MG, Natale RB, Herbst RS, Lynch TJ Jr, Prager D, Belani CP, Schiller JH, Kelly K, Spiridonidis H, Sandler A, et al: Efficacy of gefitinib, an inhibitor of the epidermal growth factor receptor tyrosine kinase, in symptomatic patients with non-small cell lung cancer: A randomized trial. JAMA 290: 2149-2158, 2003 
5. Fukuoka M, Yano S, Giaccone G, Tamura T, Nakagawa K, Douillard JY, Nishiwaki Y, Vansteenkiste J, Kudoh S, Rischin D, et al: Multi-institutional randomized phase II trial of gefitinib for previously treated patients with advanced non-small-cell lung cancer (The IDEAL 1 Trial) [corrected]. J Clin Oncol 21: 2237-2246, 2003.

6. Lynch TJ, Bell DW, Sordella R, Gurubhagavatula S, Okimoto RA Brannigan BW, Harris PL, Haserlat SM, Supko JG, Haluska FG, et al: Activating mutations in the epidermal growth factor receptor underlying responsiveness of non-small-cell lung cancer to gefitinib. N Engl J Med 350: 2129-2139, 2004.

7. Paez JG, Jänne PA, Lee JC, Tracy S, Greulich H, Gabriel S, Herman P, Kaye FJ, Lindeman N, Boggon TJ, et al: EGFR mutations in lung cancer: Correlation with clinical response to gefitinib therapy. Science 304: 1497-1500, 2004.

8. Mitsudomi T, Morita S, Yatabe Y, Negoro S, Okamoto I, Tsurutani J, Seto T, Satouchi M, Tada H, Hirashima T, et al: Gefitinib versus cisplatin plus docetaxel in patients with non-small-cell lung cancer harbouring mutations of the epidermal growth factor receptor (WJTOG3405): An open label, randomised phase 3 trial. Lancet Oncol 11: 121-128, 2010.

9. Maemondo M, Inoue A, Kobayashi K, Sugawara S, Oizumi S, Isobe H, Gemma A, Harada M, Yoshizawa H, Kinoshita I, et al: Gefitinib or chemotherapy for non-small-cell lung cancer with mutated EGFR. N Engl J Med 362: 2380-2388, 2010.

10. Therasse P, Arbuck SG, Eisenhauer EA, Wanders J, Kaplan RS, Rubinstein L, Verweij J, Van Glabbeke M, van Oosterom AT, Christian MC and Gwyther SG: New guidelines to evaluate the response to treatment in solid tumors. European Organization for Research and Treatment of Cancer, National Cancer Institute of the United States, National Cancer Institute of Canada. J Natl Cancer Inst 92: 205-216, 2000.

11. Nomoto K, Tsuta K, Takano T, Fukui T, Fukui T, Yokozawa K, Sakamoto H, Yoshida T, Maeshima AM, Shibata T, et al: Detection of EGFR mutations in archived cytologic specimens of non-small cell lung cancer using high-resolution melting analysis. Am J Clin Pathol 126: 608-615, 2006.

12. Takano T, Ohe Y, Tsuta K, Fukui T, Sakamoto H, Yoshida T, Tateishi U, Nokihara H, Yamamoto N, Sekine I, et al: Epiderma growth factor receptor mutation detection using high-resolution melting analysis predicts outcomes in patients with advanced non small cell lung cancer treated with gefitinib. Clin Cancer Res 13: 5385-5390, 2007.
13. Liu PY, Dahlberg S and Crowley J: Selection designs for pilot studies based on survival. Biometrics 49: 391-398, 1993.

14. Mok TS, Wu YL, Thongprasert S, Yang CH, Chu DT, Saijo N, Sunpaweravong P, Han B, Margono B, Ichinose Y, et al: Gefitinib or carboplatin-paclitaxel in pulmonary adenocarcinoma. N Engl J Med 361: 947-957, 2009.

15. Zhou C, Wu YL, Chen G, Feng J, Liu XQ, Wang C, Zhang S, Wang J, Zhou S, Ren S, et al: Erlotinib versus chemotherapy as first-line treatment for patients with advanced EGFR mutation-positive non-small-cell lung cancer (OPTIMAL, CTONG-0802): A multicentre, open-label, randomised, phase 3 study. Lancet Oncol 12: 735-742, 2011.

16. Rosell R, Carcereny E, Gervais R, Vergnenegre A, Massuti B, Felip E, Palmero R, Garcia-Gomez R, Pallares C, Sanchez JM, et al: Erlotinib versus standard chemotherapy as first-line treatment for European patients with advanced EGFR mutation-positive non-small-cell lung cancer (EURTAC): A multicentre, open-label, randomised phase 3 trial. Lancet Oncol 13: 239-246, 2012.

17. Yang JC, Wu YL, Schuler M, Sebastian M, Popat S, Yamamoto N, Zhou $\mathrm{C}, \mathrm{Hu} \mathrm{CP}$, O'Byrne $\mathrm{K}$, Feng J, et al: Afatinib versus cisplatin-based chemotherapy for EGFR mutation-positive lung adenocarcinoma (LUX-Lung 3 and LUX-Lung 6): Analysis of overall survival data from two randomised, phase 3 trials. Lancet Oncol 16: 141-151, 2015.

18. Walter AO, Sjin RT, Haringsma HJ, Ohashi K, Sun J, Lee K, Dubrovskiy A, Labenski M, Zhu Z, Wang Z, et al: Discovery of a mutant-selective covalent inhibitor of EGFR that overcomes T790M-mediated resistance in NSCLC. Cancer Discov 3: 1404-1415, 2013.

19. Cross DA, Ashton SE, Ghiorghiu S, Eberlein C, Nebhan CA, Spitzler PJ, Orme JP, Finlay MR, Ward RA, Mellor MJ, et al: AZD9291, an irreversible EGFR TKI, overcomes T790M-mediated resistance to EGFR inhibitors in lung cancer. Cancer Discov 4: 1046-1061, 2014.

20. Jiang T and Zhou C: Clinical activity of the mutant-selective EGFR inhibitor AZD9291 in patients with EGFR inhibitor-resistant non-small cell lung cancer. Transl Lung Cancer Res 3: 370-372, 2014. 